\title{
On-chip Microwave to Optical Transduction Using Rare Earth Doped Materials
}

\author{
Andrei Faraon*, Jake Rochman, Tian Xie, John G. Bartholomew \\ T. J. Watson Laboratory of Applied Physics, California Institute of Technology, 1200 E. California Blvd., Pasadena, CA 91125, USA \\ Kavli Nanoscience Institute, California Institute of Technology, Pasadena, CA, 91125, USA \\ Institute for Quantum Information and Matter, California Institute of Technology, Pasadena, CA, 91125, USA \\ *faraon@caltech.edu
}

\begin{abstract}
Rare-earth ions simultaneously coupled to optical and microwave resonators are one of the platforms for realizing efficient microwave to optical quantum transducers. I discuss our group's recent progress towards this goal. @ 2021 The Author(s)

OCIS codes: 270.0270 Quantum optics; 190.0190 Nonlinear optics
\end{abstract}

Rare-earth ions in solids exhibit transitions both in the microwave domain and optical domain that can be used to mediate conversion between these frequencies. In order for the transduction to be efficient, it is necessary for the ions to be coupled to both optical and microwave resonators, while the energy difference between the optical and the microwave photons is supplied by an optical pump beam[1]. In order for the optical pump beam to be kept at power levels compatible with current dilution refrigerators, it is important for the transduction devices to be made on-chip. In this presentation I will give an overview of our recent work towards on-chip microwave to optical transduction using ytterbium[2] and erbium doped materials. These devices integrate photonic crystal resonators and superconducting microwave resonators and are probed at dilution refrigerator temperatures. I present the observed transduction signals and discuss the current limitations in device efficiency and the path to improve it.

1. Williamson, L.A., Y.H. Chen, and J.J. Longdell, Magneto-optic modulator with unit quantum efficiency. Phys Rev Lett, 2014.

113(20): p. 203601.

2. Bartholomew, J.G., et al., On-chip coherent microwave-to-optical transduction mediated by ytterbium in YVO4. Nature

Communications, 2020. 11(1): p. 3266. 